\title{
Optimal Transport and Ricci Curvature for Metric-Measure Spaces
}

\author{
John Lott \\ Abstract. We survey work of Lott-Villani and Sturm on lower \\ Ricci curvature bounds for metric-measure spaces.
}

An intriguing question is whether one can extend notions of smooth Riemannian geometry to general metric spaces. Besides the inherent interest, such extensions sometimes allow one to prove results about smooth Riemannian manifolds, using compactness theorems.

There is a good notion of a metric space having "sectional curvature bounded below by $K$ " or "sectional curvature bounded above by $K$ ", due to Alexandrov. We refer to the articles of Petrunin and Buyalo-Schroeder in this volume for further information on these two topics. In this article we address the issue of whether there is a good notion of a metric space having "Ricci curvature bounded below by $K$ ".

A motivation for this question comes from Gromov's precompactness theorem [14, Theorem 5.3]. Let $\mathcal{M}$ denote the set of compact metric spaces (modulo isometry) with the Gromov-Hausdorff topology. The precompactness theorem says that given $N \in \mathbb{Z}^{+}, D<\infty$ and $K \in \mathbb{R}$, the subset of $\mathcal{M}$ consisting of closed Riemannian manifolds $(M, g)$ with $\operatorname{dim}(M)=N$, Ric $\geq K g$ and diam $\leq D$, is precompact. The limit points in $\mathcal{M}$ of this subset will be metric spaces of Hausdorff dimension at most $N$, but generally are not manifolds. However, one would like to say that in some generalized sense they do have Ricci curvature bounded below by $K$. Deep results about the structure of such limit points, which we call Ricci limits, were obtained by Cheeger and Colding $[\mathbf{8}, \mathbf{9}, \mathbf{1 0}]$. We refer to the article of Guofang Wei in this volume for further information.

In the work of Cheeger and Colding, and in earlier work of Fukaya [13], it turned out to be useful to consider not just metric spaces, but rather metric spaces equipped with measures. Given a compact metric space $(X, d)$, let

The author was partially supported by NSF grant DMS-0604829 during the writing of this article. 
$P(X)$ denote the set of Borel probability measures on $X$. That is, $\nu \in P(X)$ means that $\nu$ is a nonnegative Borel measure on $X$ with $\int_{X} d \nu=1$. We put the weak-* topology on $P(X)$, so $\lim _{i \rightarrow \infty} \nu_{i}=\nu$ if and only if for all $f \in C(X)$, we have $\lim _{i \rightarrow \infty} \int_{X} f d \nu_{i}=\int_{X} f d \nu$. Then $P(X)$ is compact.

Definition 0.1. A compact metric measure space is a triple $(X, d, \nu)$ where $(X, d)$ is a compact metric space and $\nu \in P(X)$.

Definition 0.2. Given two compact metric spaces $\left(X_{1}, d_{1}\right)$ and $\left(X_{2}, d_{2}\right)$, an $\epsilon$-Gromov-Hausdorff approximation from $X_{1}$ to $X_{2}$ is a (not necessarily continuous) map $f: X_{1} \rightarrow X_{2}$ so that

(i) For all $x_{1}, x_{1}^{\prime} \in X_{1},\left|d_{2}\left(f\left(x_{1}\right), f\left(x_{1}^{\prime}\right)\right)-d_{1}\left(x_{1}, x_{1}^{\prime}\right)\right| \leq \epsilon$.

(ii) For all $x_{2} \in X_{2}$, there is an $x_{1} \in X_{1}$ so that $d_{2}\left(f\left(x_{1}\right), x_{2}\right) \leq \epsilon$.

A sequence $\left\{\left(X_{i}, d_{i}, \nu_{i}\right)\right\}_{i=1}^{\infty}$ of compact metric-measure spaces converges to $(X, d, \nu)$ in the measured Gromov-Hausdorff topology if there are Borel $\epsilon_{i}$-approximations $f_{i}: X_{i} \rightarrow X$, with $\lim _{i \rightarrow \infty} \epsilon_{i}=0$, so that $\lim _{i \rightarrow \infty}\left(f_{i}\right)_{*} \nu_{i}=$ $\nu$ in $P(X)$.

REMARK 0.3. There are other interesting topologies on the set of metricmeasure spaces, discussed in $\left[\mathbf{1 4}\right.$, Chapter $\left.3 \frac{1}{2}\right]$.

If $M$ is a compact manifold with Riemannian metric $g$ then we also let $(M, g)$ denote the underlying metric space. There is a canonical probability measure on $M$ given by the normalized volume form $\frac{\operatorname{dvol}_{M}}{\operatorname{vol}(M)}$. One can easily extend Gromov's precompactness theorem to say that given $N \in \mathbb{Z}^{+}, D<\infty$ and $K \in \mathbb{R}$, the triples $\left(M, g, \frac{\operatorname{dvol}_{M}}{\operatorname{vol}(M)}\right)$ with $\operatorname{dim}(M)=N$, Ric $\geq K g$ and diam $\leq D$ form a precompact subset in the measured Gromov-Hausdorff (MGH) topology. The limit points of this subset are now metric-measure spaces $(X, d, \nu)$. One would like to say that they have "Ricci curvature bounded below by $K$ " in some generalized sense.

The metric space $(X, d)$ of a Ricci limit is necessarily a length space. Hereafter we mostly restrict our attention to length spaces. So the question that we address is whether there is a good notion of a compact measured length space $(X, d, \nu)$ having "Ricci curvature bounded below by $K$ ". The word "good" is a bit ambiguous here, but we would like our definition to have the following properties.

WISHLIST 0.4 .

1. If $\left\{\left(X_{i}, d_{i}, \nu_{i}\right)\right\}_{i=1}^{\infty}$ is a sequence of compact measured length spaces with "Ricci curvature bounded below by $K$ " and $\lim _{i \rightarrow \infty}\left(X_{i}, d_{i}, \nu_{i}\right)=$ $(X, d, \nu)$ in the measured Gromov-Hausdorff topology then $(X, d, \nu)$ has "Ricci curvature bounded below by $K$ ".

2. If $(M, g)$ is a compact Riemannian manifold then the triple $\left(M, g, \frac{\operatorname{dvol}_{M}}{\operatorname{vol}(M)}\right)$ has "Ricci curvature bounded below by $K$ " if and only if Ric $\geq K g$ in the usual sense. 
3. One can prove some nontrivial results about measured length spaces having "Ricci curvature bounded below by $K$ ".

It is not so easy to come up with a definition that satisfies all of these properties. One possibility would be to say that $(X, d, \nu)$ has "Ricci curvature bounded below by $K$ " if and only if it is an MGH limit of Riemannian manifolds with Ric $\geq K g$, but this is a bit tautological. We want instead a definition that depends in an intrinsic way on $(X, d, \nu)$. We refer to [8, Appendix 2] for further discussion of the problem.

In fact, it will turn out that we will want to specify an effective dimension $N$, possibly infinite, of the measured length space. That is, we want to define a notion of $(X, d, \nu)$ having " $N$-Ricci curvature bounded below by $K$ ", where $N$ is a parameter that is part of the definition. The need to input the parameter $N$ can be seen from the Bishop-Gromov inequality for complete $n$-dimensional Riemannian manifolds with nonnegative Ricci curvature. It says that $r^{-n} \operatorname{vol}\left(B_{r}(m)\right)$ is nonincreasing in $r$, where $B_{r}(m)$ is the $r$-ball centered at $m$. We will want a Bishop-Gromov-type inequality to hold in the length space setting, but when we go from manifolds to length spaces there is no a priori value for the parameter $n$. Hence for each $N \in[1, \infty]$, there will be a notion of $(X, d, \nu)$ having " $N$-Ricci curvature bounded below by $K$ ".

The goal now is to find some property which we know holds for $N$ dimensional Riemannian manifolds with Ricci curvature bounded below, and turn it into a definition for measured length spaces. A geometer's first inclination may be to just use the Bishop-Gromov inequality, at least if $N<\infty$, for example to say that $(X, d, \nu)$ has "nonnegative $N$-Ricci curvature" if and only if for each $x \in \operatorname{supp}(\nu), r^{-N} \nu\left(B_{r}(x)\right)$ is nonincreasing in $r$. Although this is the simplest possibility, it turns out that it is not satisfactory; see Remark 4.9. Instead, we will derive a Bishop-Gromov inequality as part of a more subtle definition.

The definition that we give in this paper may seem to come from left field, at least from the viewpoint of standard geometry. It comes from a branch of applied mathematics called optimal transport, which can be informally considered to be the study of moving dirt around. The problem originated with Monge in the paper [27], whose title translates into English as "On the theory of excavations and fillings". (In that paper Monge also introduced the idea of a line of curvature of a surface.) The problem that Monge considered was how to transport a "before" dirtpile to an "after" dirtpile with minimal total "cost", where he took the cost of transporting a unit mass of dirt between points $x$ and $y$ to be $d(x, y)$. Such a transport $F: X \rightarrow X$ is called a Monge transport. An account of Monge's life, and his unfortunate political choices, is in $[\mathbf{4}]$.

Since Monge's time, there has been considerable work on optimal transport. Of course, the original case of interest was optimal transport on Euclidean space. Kantorovich introduced a important relaxation of Monge's 
original problem, in which not all of the dirt from a given point $x$ has to go to a single point $y$. That is, the dirt from $x$ is allowed to be spread out over the space. Kantorovich showed that there is always an optimal transport scheme in his sense. (Kantorovich won a 1975 Nobel Prize in economics.) We refer to the book [38] for a lively and detailed account of optimal transport.

In Section 1 we summarize some optimal transport results from a modern perspective. We take the cost function of transporting a unit mass of dirt to be $d(x, y)^{2}$ instead of Monge's cost function $d(x, y)$. The relation to Ricci curvature comes from work of Otto-Villani $[\mathbf{3 0}]$ and Cordero-ErausquinMcCann-Schmuckenschläger [11]. They showed that optimal transport on a Riemannian manifold is affected by the Ricci tensor. To be a bit more precise, the Ricci curvature affects the convexity of certain entropy functionals along an optimal transport path. Details are in Section 2.

The idea now, implemented independently by Lott-Villani and Sturm, is to define the property " $N$-Ricci curvature bounded below by $K$ ", for a measured length space $(X, d, \nu)$, in terms of the convexity of certain entropy functionals along optimal transport paths in the auxiliary space $P(X)$. We present the definition and its initial properties in Section 3. We restrict in that section to the case $K=0$, where the discussion becomes a bit simpler. We show that Condition 1. from Wishlist 0.4 is satisfied. In Section 4 we show that Condition 2. from Wishlist 0.4 is satisfied. In Section 5 we give the definition of $(X, d, \nu)$ having $N$-Ricci curvature bounded below by $K$, for $K \in \mathbb{R}$.

Concerning Condition 3. of the Wishlist, in Sections 3, 4 and 5 we give some geometric results that one can prove about measured length spaces with Ricci curvature bounded below. In particular, there are applications to Ricci limit spaces. In Section 6 we give some analytic results. In Section 7 we discuss some further issues.

We mostly focus on results from [23] and [24], mainly because of the author's familiarity with those papers. However, we emphasize that many parallel results were obtained independently by Karl-Theodor Sturm in [36, 37]. Background information on optimal transport is in $[\mathbf{3 8}]$ and $[\mathbf{3 9}]$. The latter book also contains a more detailed exposition of some of the topics of this survey.

I thank Cédric Villani for an enjoyable collaboration.

\section{Optimal transport}

Let us state the Kantorovich transport problem. We take $(X, d)$ to be a compact metric space. Our "before" and "after" dirtpiles are measures $\mu_{0}, \mu_{1} \in P(X)$. They both have mass one. We want to move the total amount of dirt from $\mu_{0}$ to $\mu_{1}$ most efficiently. A moving scheme, maybe not optimal, will be called a transference plan. Intuitively, it amounts to specifying how much dirt is moved from a point $x_{0}$ to a point $x_{1}$. That is, we have a probability measure $\pi \in P(X \times X)$, which we informally write as $\pi\left(x_{0}, x_{1}\right)$. The 
statement that $\pi$ does indeed transport $\mu_{0}$ to $\mu_{1}$ translates to the condition that

$$
\left(p_{0}\right)_{*} \pi=\mu_{0}, \quad\left(p_{1}\right)_{*} \pi=\mu_{1},
$$

where $p_{0}, p_{1}: X \times X \rightarrow X$ are projections onto the first and second factors, respectively.

We will use optimal transport with quadratic cost function (square of the distance). The total cost of the transference plan $\pi$ is given by adding the contributions of $d\left(x_{0}, x_{1}\right)^{2}$ with respect to $\pi$. Taking the infimum of this with respect to $\pi$ gives the square of the Wasserstein distance $W_{2}\left(\mu_{0}, \mu_{1}\right)$ between $\mu_{0}$ and $\mu_{1}$, i.e.,

$$
W_{2}\left(\mu_{0}, \mu_{1}\right)^{2}=\inf _{\pi} \int_{X \times X} d\left(x_{0}, x_{1}\right)^{2} d \pi\left(x_{0}, x_{1}\right)
$$

where $\pi$ ranges over the set of all transference plans between $\mu_{0}$ and $\mu_{1}$. Any minimizer $\pi$ for this variational problem is called an optimal transference plan.

In (1.2), one can replace the infimum by the minimum [38, Proposition $2.1]$, i.e., there always exists (at least) one optimal transference plan. It turns out that $W_{2}$ is a metric on $P(X)$. The topology that it induces on $P(X)$ is the weak-* topology [38, Theorems 7.3 and 7.12]. When equipped with the metric $W_{2}, P(X)$ is a compact metric space. In this way, to each compact metric space $X$ we have assigned another compact metric space $P(X)$. The Wasserstein space $\left(P(X), W_{2}\right)$ seems to be a very natural object in mathematics. It generally has infinite topological or Hausdorff dimension. (If $X$ is a finite set then $P(X)$ is a simplex, with a certain metric.) It is always contractible, as can be seen by fixing a measure $\mu_{0} \in P(X)$ and linearly contracting other measures $\mu \in P(X)$ to $\mu_{0}$ by $t \rightarrow t \mu_{0}+(1-t) \mu$.

Proposition 1.3 ([23, Corollary 4.3]). If $\lim _{i \rightarrow \infty}\left(X_{i}, d_{i}\right)=(X, d)$ in the Gromov-Hausdorff topology then $\lim _{i \rightarrow \infty}\left(P\left(X_{i}\right), W_{2}\right)=\left(P(X), W_{2}\right)$ in the Gromov-Hausdorff topology.

A Monge transport is a transference plan coming from a map $F: X \rightarrow X$ with $F_{*} \mu_{0}=\mu_{1}$, given by $\pi=(\mathrm{Id}, F)_{*} \mu_{0}$. In general an optimal transference plan does not have to be a Monge transport, although this may be true under some assumptions.

What does optimal transport look like in Euclidean space $\mathbb{R}^{n}$ ? Suppose that $\mu_{0}$ and $\mu_{1}$ are compactly supported and absolutely continuous with respect to Lebesgue measure. Brenier [5] and Rachev-Rüschendorf [33] showed that there is a unique optimal transference plan between $\mu_{0}$ and $\mu_{1}$, which is a Monge transport. Furthermore, there is a convex function $V$ on $\mathbb{R}^{n}$ so that for almost all $\vec{x}$, the Monge transport is given by $F(\vec{x})=\vec{\nabla}_{\vec{x}} V$. So to find the optimal transport, one finds a convex function $V$ such that the pushforward, under the map $\nabla V: \mathbb{R}^{n} \rightarrow \mathbb{R}^{n}$, sends $\mu_{0}$ to $\mu_{1}$. This solves 
the Monge problem for such measures, under our assumption of quadratic cost function. The solution to the original problem of Monge, with linear cost function, is more difficult; see [12].

The statement of the Brenier-Rachev-Rüschendorf theorem may sound like anathema to a geometer. One is identifying the gradient of $V$ (at $\vec{x}$ ), which is a vector, with the image of $\vec{x}$ under a map, which is a point. Because of this, it is not evident how to extend even the statement of the theorem if one wants to do optimal transport on a Riemannian manifold. The extension was done by McCann $[\mathbf{2 6}]$. The key point is that on $\mathbb{R}^{n}$, we can write $\vec{\nabla}_{\vec{x}} V=\vec{x}-\vec{\nabla}_{\vec{x}} \phi$, where $\phi(\vec{x})=\frac{|\vec{x}|^{2}}{2}-V(\vec{x})$. To understand the relation between $V$ and $\phi$, we note that if the convex function $V$ were smooth then $\phi$ would have Hessian bounded above by the identity. On a Riemannian manifold $(M, g)$, McCann's theorem says that an optimal transference plan between two compactly supported absolutely continuous measures is a Monge transport $F$ that satisfies $F(m)=\exp _{m}\left(-\nabla_{m} \phi\right)$ for almost all $m$, where $\phi$ is a function on $M$ with Hessian bounded above by $g$ in a generalized sense. More precisely, $\phi$ is $\frac{d^{2}}{2}$-concave in the sense that it can be written in the form

$$
\phi(m)=\inf _{m^{\prime} \in M}\left(\frac{d\left(m, m^{\prime}\right)^{2}}{2}-\widetilde{\phi}\left(m^{\prime}\right)\right)
$$

for some function $\widetilde{\phi}: M \rightarrow[-\infty, \infty)$.

Returning to the metric space setting, if $(X, d)$ is a compact length space and one has an optimal transference plan $\pi$ then one would physically perform the transport by picking up pieces of dirt in $X$ and moving them along minimal geodesics to other points in $X$, in a way consistent with the transference plan $\pi$. The transference plan $\pi$ tells us how much dirt has to go from $x_{0}$ to $x_{1}$, but does not say anything about which minimal geodesics from $x_{0}$ to $x_{1}$ we should actually use. After making such a choice of minimizing geodesics, we obtain a 1-parameter family of measures $\left\{\mu_{t}\right\}_{t \in[0,1]}$ by stopping the physical transport procedure at time $t$ and looking at where the dirt is. This suggests looking at $\left(P(X), W_{2}\right)$ as a length space.

Proposition 1.5 ([23, Corollary 2.7], [36, Proposition 2.10(iii)]). If $(X, d)$ is a compact length space then $\left(P(X), W_{2}\right)$ is a compact length space.

Hereafter we assume that $(X, d)$ is a compact length space. By definition, a Wasserstein geodesic is a minimizing geodesic in the length space $\left(P(X), W_{2}\right)$. (We will always parametrize minimizing geodesics in length spaces to have constant speed.) The length space $\left(P(X), W_{2}\right)$ has some interesting features; even for simple $X$, there may be an uncountable number of Wasserstein geodesics between two measures $\mu_{0}, \mu_{1} \in P(X)[\mathbf{2 3}$, Example 2.9].

As mentioned above, there is a relation between minimizing geodesics in $\left(P(X), W_{2}\right)$ and minimizing geodesics in $X$. Let $\Gamma$ be the set of minimizing 
geodesics $\gamma:[0,1] \rightarrow X$. It is compact in the uniform topology. For any $t \in[0,1]$, the evaluation map $e_{t}: \Gamma \rightarrow X$ defined by

$$
e_{t}(\gamma)=\gamma(t)
$$

is continuous. Let $E: \Gamma \rightarrow X \times X$ be the "endpoints" map given by $E(\gamma)=$ $\left(e_{0}(\gamma), e_{1}(\gamma)\right)$. A dynamical transference plan consists of a transference plan $\pi$ and a Borel measure $\Pi$ on $\Gamma$ such that $E_{*} \Pi=\pi$; it is said to be optimal if $\pi$ itself is. In words, the transference plan $\pi$ tells us how much mass goes from a point $x_{0}$ to another point $x_{1}$, but does not tell us about the actual path that the mass has to follow. Intuitively, mass should flow along geodesics, but there may be several possible choices of geodesics between two given points and the transport may be divided among these geodesics; this is the information provided by $\Pi$.

If $\Pi$ is an optimal dynamical transference plan then for $t \in[0,1]$, we put

$$
\mu_{t}=\left(e_{t}\right)_{*} \Pi \text {. }
$$

The one-parameter family of measures $\left\{\mu_{t}\right\}_{t \in[0,1]}$ is called a displacement interpolation. In words, $\mu_{t}$ is what has become of the mass of $\mu_{0}$ after it has travelled from time 0 to time $t$ according to the dynamical transference plan $\Pi$.

Proposition 1.8 ([23, Lemma 2.4 and Proposition 2.10]). Any displacement interpolation is a Wasserstein geodesic. Conversely, any Wasserstein geodesic arises as a displacement interpolation from some optimal dynamical transference plan.

In the Riemannian case, if $\mu_{0}, \mu_{1}$ are absolutely continuous with respect to $\mathrm{dvol}_{M}$, and $F(m)=\exp _{m}\left(-\nabla_{m} \phi\right)$ is the Monge transport between them, then there is a unique Wasserstein geodesic between $\mu_{0}$ and $\mu_{1}$ given by $\mu_{t}=\left(F_{t}\right)_{*} \mu_{0}$, where $F_{t}(m)=\exp _{m}\left(-t \nabla_{m} \phi\right)$. Here $\mu_{t}$ is also absolutely continuous with respect to $\mathrm{dvol}_{M}$. On the other hand, if $\mu_{0}=\delta_{m_{0}}$ and $\mu_{1}=\delta_{m_{1}}$ then any Wasserstein geodesic from $\mu_{0}$ to $\mu_{1}$ is of the form $\mu_{t}=$ $\delta_{c(t)}$, where $c$ is a minimizing geodesic from $m_{0}$ to $m_{1}$. In particular, the Wasserstein geodesic need not be unique.

In a remarkable paper [29], motivated by PDE problems, Otto constructed a formal infinite-dimensional Riemannian metric $g_{H^{-1}}$ on $P\left(\mathbb{R}^{n}\right)$. To describe $g_{H^{-1}}$, for simplicity we work with a compact Riemannian manifold $M$ instead of $\mathbb{R}^{n}$. Suppose that $\mu \in P(M)$ can be written as $\mu=\rho \operatorname{dvol}_{M}$, with $\rho$ a smooth positive function. We formally think of a tangent vector $\delta \mu \in T_{\mu} P(M)$ as being a variation of $\mu$, which we take to be $(\delta \rho) \operatorname{dvol}_{M}$ with $\delta \rho \in C^{\infty}(M)$. There is a $\Phi \in C^{\infty}(M)$, unique up to constants, so that $\delta \rho=d^{*}(\rho d \Phi)$. Then by definition,

$$
g_{H^{-1}}(\delta \mu, \delta \mu)=\int_{M}|d \Phi|^{2} d \mu .
$$


One sees that in terms of $\delta \rho \in C^{\infty}(M), g_{H^{-1}}$ corresponds to a weighted $H^{-1}$-inner product.

Otto showed that the corresponding distance function on $P(M)$ is formally $W_{2}$, and that the "infinite-dimensional Riemannian manifold" $\left(P\left(\mathbb{R}^{n}\right), g_{H^{-1}}\right)$ formally has nonnegative sectional curvature. One can make rigorous sense of these statements in terms of Alexandrov geometry.

Proposition 1.10 ([23, Theorem A.8], [36, Proposition $2.10($ iv) $]$ ). $\left(P(M), W_{2}\right)$ has nonnegative Alexandrov curvature if and only if $M$ has nonnegative sectional curvature.

Proposition 1.11 ([23, Proposition A.33]). If $M$ has nonnegative sectional curvature then for each absolutely continuous measure $\mu=\rho \operatorname{dvol}_{M} \in$ $P(M)$, the tangent cone $T_{\mu} P(M)$ is an inner product space. If $\rho$ is smooth and positive then the inner product on $T_{\mu} P(M)$ equals $g_{H^{-1}}$.

An open question is whether there is any good sense in which $\left(P(M), W_{2}\right)$, or a large part thereof, carries an infinite-dimensional Riemannian structure. The analogous question for finite-dimensional Alexandrov spaces has been much studied.

REMARK 1.12. In Sturm's work he uses the following interesting metric $D$ on the set of compact metric-measure spaces [36, Definition 3.2]. Given $\mathcal{X}_{1}=\left(X_{1}, d_{1}, \nu_{1}\right)$ and $\mathcal{X}_{2}=\left(X_{2}, d_{2}, \nu_{2}\right)$, let $\widehat{d}$ denote a metric on the disjoint union $X_{1} \amalg X_{2}$ such that $\left.\widehat{d}\right|_{X_{1} \times X_{1}}=d_{1}$ and $\left.\widehat{d}\right|_{X_{2} \times X_{2}}=d_{2}$. Then

$$
D\left(\mathcal{X}_{1}, \mathcal{X}_{2}\right)^{2}=\inf _{\widehat{d}, q} \int_{X_{1} \times X_{2}} \widehat{d}\left(x_{1}, x_{2}\right)^{2} d q\left(x_{1}, x_{2}\right)
$$

where $q$ runs over probability measures on $X_{1} \times X_{2}$ whose pushforwards onto $X_{1}$ and $X_{2}$ are $\nu_{1}$ and $\nu_{2}$, respectively. If one restricts to metric-measure spaces with an upper diameter bound whose measures have full support and satisfy a uniform doubling condition (which will be the case with a lower Ricci curvature bound) then the topology coming from $D$ coincides with the MGH topology of Definition 0.2 [36, Lemma 3.18], [39].

\section{Motivation for displacement convexity}

To say a bit more about the PDE motivation, we recall that the heat equation $\frac{\partial f}{\partial t}=\nabla^{2} f$ can be considered to be the formal gradient flow of the Dirichlet energy $E(f)=\frac{1}{2} \int_{M}|d f|^{2} \operatorname{dvol}_{M}$ on $L^{2}\left(M, \operatorname{dvol}_{M}\right)$. (Our conventions are that a function decreases along the flowlines of its gradient flow, so on a finite-dimensional Riemannian manifold $Y$ the gradient flow of a function $F \in C^{\infty}(Y)$ is $\frac{d c}{d t}=-\nabla F$.) Jordan-Kinderlehrer-Otto showed that the heat equation on measures can also be formally written as a gradient flow [16]. Namely, for a smooth probability measure $\mu=\rho \frac{\operatorname{dvol}_{M}}{\operatorname{vol}(M)}$, let us 
put $H_{\infty}(\mu)=\int_{M} \rho \log \rho \frac{\operatorname{dvol}_{M}}{\operatorname{vol}(M)}$. Then the heat equation $\frac{\partial}{\partial t}\left(\rho \frac{\operatorname{dvol}_{M}}{\operatorname{vol}(M)}\right)=$ $\nabla^{2} \rho \frac{\operatorname{dvol}_{M}}{\operatorname{vol}(M)}$ is formally the gradient flow of $H_{\infty}$ on $P(M)$, where $P(M)$ has Otto's formal Riemannian metric. Identifying a.c. measures and measurable functions using $\frac{\operatorname{dvol}_{M}}{\operatorname{vol}(M)}$, this gave a new way to realize the heat equation as a gradient flow.

Although this approach may not give much new information about the heat equation, it has more relevance if one considers other functions $H$ on $P(M)$, whose gradient flows can give rise to interesting nonlinear PDE's such as the porous medium equation. Again formally, if one has positive lower bounds on the Hessian of $H$ then one can draw conclusions about uniqueness of critical points and rates of convergence of the gradient flow to the critical point, which one can then hope to make rigorous. This reasoning motivated McCann's notion of displacement convexity, i.e., convexity of a function $H$ along Wasserstein geodesics [25]. (We recall that on a smooth manifold, a smooth function has a nonnegative Hessian if and only if it is convex when restricted to each geodesic.)

In a related direction, Otto and Villani [30] saw that convexity properties on $P(M)$ could be used to give heuristic arguments for functional inequalities on $M$, such as the log Sobolev inequality. They could then give rigorous proofs based on these heuristic arguments. Given a smooth background probability measure $\nu=e^{-\Psi} \operatorname{dvol}_{M}$ and an absolutely continuous probability measure $\mu=\rho \nu$, let us now put $H_{\infty}(\mu)=\int_{M} \rho(\log \rho) d \nu$. As part of their work, Otto and Villani computed the formal Hessian of the function $H_{\infty}$ on $P(M)$ and found that it is bounded below by $K g_{H^{-1}}$ provided that the Bakry-Émery tensor $\operatorname{Ric}_{\infty}=\operatorname{Ric}+\operatorname{Hess}(\Psi)$ satisfies $\operatorname{Ric}_{\infty} \geq K g$ on $M$. This was perhaps the first indication that Ricci curvature is related to convexity properties on Wasserstein space.

Around the same time, Cordero-Erausquin-McCann-Schmuckenschläger [11] gave a rigorous proof of the convexity of certain functions on $P(M)$ when $M$ has dimension $n$ and nonnegative Ricci curvature. Suppose that $A:[0, \infty) \rightarrow \mathbb{R}$ is a continuous convex function with $A(0)=0$ such that $\lambda \rightarrow \lambda^{n} A\left(\lambda^{-n}\right)$ is a convex function on $\mathbb{R}^{+}$. If $\mu=\rho \frac{\operatorname{dvol}_{M}}{\operatorname{vol}(M)}$ is an absolutely continuous probability measure then put $H_{A}(\mu)=\int_{M} A(\rho) \frac{\operatorname{dvol}_{M}}{\operatorname{vol}(M)}$. The statement is that if $\mu_{0}, \mu_{1} \in P(M)$ are absolutely continuous, and $\left\{\mu_{t}\right\}_{t \in[0,1]}$ is the (unique) Wasserstein geodesic between them, then $H_{A}\left(\mu_{t}\right)$ is convex in $t$, again under the assumption of nonnegative Ricci curvature.

Finally, von Renesse and Sturm [35] extended the work of CorderoErausquin-McCann-Schmuckenschläger to show that the function $H_{\infty}$, defined by $H_{\infty}\left(\rho \operatorname{dvol}_{M}\right)=\int_{M} \rho \log \rho \frac{\operatorname{dvol}_{M}}{\operatorname{vol}(M)}$, is $K$-convex along Wasserstein geodesics between absolutely-continuous measures if and only if Ric $\geq K g$. (The relation with the Otto-Villani result is that $\Psi$ is taken to be constant, so $\nu=\frac{\operatorname{dvol}_{M}}{\operatorname{vol}(M)}$.) The "if" implication is along the lines of the CorderoErausquin-McCann-Schmuckenschläger result and the "only if" implication involves some local arguments. 
Although these results indicate a formal relation between Ricci curvature and displacement convexity, one can ask for a more intuitive understanding. Here is one example.

EXAMPLE 2.1. Consider the functional $H_{\infty}\left(\rho \frac{\mathrm{dvol}_{M}}{\operatorname{vol}(M)}\right)=\int_{M} \rho \log \rho \frac{\operatorname{dvol}_{M}}{\operatorname{vol}(M)}$. It is minimized, among absolutely continuous probability measures on $M$, when $\rho=1$, i.e., when the measure $\mu=\rho \frac{\operatorname{dvol}_{M}}{\operatorname{vol}(M)}$ is the uniform measure $\frac{\operatorname{dvol}_{M}}{\operatorname{vol}(M)}$. In this sense, $H_{\infty}$ measures the nonuniformity of $\mu$ with respect to $\frac{\operatorname{dvol}_{M}}{\operatorname{vol}(M)}$. Now take $M=S^{2}$. Let $\mu_{0}$ and $\mu_{1}$ be two small congruent rotationally symmetric blobs, centered at the north and south poles respectively. Clearly $U_{\infty}\left(\mu_{0}\right)=U_{\infty}\left(\mu_{1}\right)$. Consider the Wasserstein geodesic from $\mu_{0}$ to $\mu_{1}$. It takes the blob $\mu_{0}$ and pushes it down in a certain way along the lattitudes until it becomes $\mu_{1}$. At an intermediate time, say around $t=\frac{1}{2}$, the blob has spread out to form a ring. When it spreads, it becomes more uniform with respect to $\frac{\operatorname{dvol}_{M}}{\operatorname{vol}(M)}$. Thus the nonuniformity at an intermediate time is at most that at times $t=0$ or $t=1$. This can be seen as a consequence of the convexity of $H_{\infty}\left(\mu_{t}\right)$ in $t$, i.e., for $t \in[0,1]$ we have $H_{\infty}\left(\mu_{t}\right) \leq H_{\infty}\left(\mu_{0}\right)=H_{\infty}\left(\mu_{1}\right)$. In this way the displacement convexity of $H_{\infty}$ can be seen as an averaged form of the focusing property of positive curvature. Of course this example does not indicate why the relevant curvature is Ricci curvature, as opposed to some other curvature, but perhaps gives some indication of why curvature is related to displacement convexity.

\section{Entropy functions and displacement convexity}

In this section we give the definition of nonnegative $N$-Ricci curvature. We then outline the proof that it is preserved under measured GromovHausdorff limits. In the next section we relate the definition to the classical notion of Ricci curvature, in the case of a smooth metric-measure space.

3.1. Definitions. We first define the relevant "entropy" functionals.

Let $X$ be a compact Hausdorff space. Let $U:[0, \infty) \rightarrow \mathbb{R}$ be a continuous convex function with $U(0)=0$. Given a reference probability measure $\nu \in$ $P(X)$, define the entropy function $U_{\nu}: P(X) \rightarrow \mathbb{R} \cup\{\infty\}$ by

$$
U_{\nu}(\mu)=\int_{X} U(\rho(x)) d \nu(x)+U^{\prime}(\infty) \mu_{s}(X),
$$

where

$$
\mu=\rho \nu+\mu_{s}
$$

is the Lebesgue decomposition of $\mu$ with respect to $\nu$ into an absolutely continuous part $\rho \nu$ and a singular part $\mu_{s}$, and

$$
U^{\prime}(\infty)=\lim _{r \rightarrow \infty} \frac{U(r)}{r} .
$$


ExAmple 3.4. Given $N \in(1, \infty]$, take the function $U_{N}$ on $[0, \infty)$ to be

$$
U_{N}(r)= \begin{cases}N r\left(1-r^{-1 / N}\right) & \text { if } 1<N<\infty \\ r \log r & \text { if } N=\infty\end{cases}
$$

Let $H_{N, \nu}: P(X) \rightarrow \mathbb{R} \cup\{\infty\}$ be the corresponding entropy function. If $N \in(1, \infty)$ then

$$
H_{N, \nu}=N-N \int_{X} \rho^{1-\frac{1}{N}} d \nu
$$

while if $N=\infty$ then

$$
H_{\infty, \nu}(\mu)=\int_{X} \rho \log \rho d \nu
$$

if $\mu$ is absolutely continuous with respect to $\nu$ and $H_{\infty, \nu}(\mu)=\infty$ otherwise.

One can show that as a function of $\mu \in P(X), U_{\nu}(\mu)$ is minimized when $\mu=\nu$. It would be better to call $U_{\nu}$ a "negative entropy", but we will be sloppy. Here are the technical properties of $U_{\nu}$ that we need.

Proposition 3.8 ([21], [23, Theorem B.33]).

(i) $U_{\nu}(\mu)$ is a lower semicontinuous function of $(\mu, \nu) \in P(X) \times P(X)$. That is, if $\left\{\mu_{k}\right\}_{k=1}^{\infty}$ and $\left\{\nu_{k}\right\}_{k=1}^{\infty}$ are sequences in $P(X)$ with $\lim _{k \rightarrow \infty} \mu_{k}=\mu$ and $\lim _{k \rightarrow \infty} \nu_{k}=\nu$ in the weak-* topology then

$$
U_{\nu}(\mu) \leq \liminf _{k \rightarrow \infty} U_{\nu_{k}}\left(\mu_{k}\right)
$$

(ii) $U_{\nu}(\mu)$ is nonincreasing under pushforward. That is, if $Y$ is a compact Hausdorff space and $f: X \rightarrow Y$ is a Borel map then

$$
U_{f_{*} \nu}\left(f_{*} \mu\right) \leq U_{\nu}(\mu)
$$

In fact, the $U^{\prime}(\infty) \mu_{s}(X)$ term in (3.1) is dictated by the fact that we want $U_{\nu}$ to be lower semicontinuous on $P(X)$.

We now pass to the setting of a compact measured length space $(X, d, \nu)$. The definition of nonnegative $N$-Ricci curvature will be in terms of the convexity of certain entropy functions on $P(X)$, where the entropy is relative to the background measure $\nu$. By "convexity" we mean convexity along Wasserstein geodesics, i.e., displacement convexity. We first describe the relevant class of entropy functions.

If $N \in[1, \infty)$ then we define $\mathcal{D} \mathcal{C}_{N}$ to be the set of such functions $U$ so that the function

$$
\psi(\lambda)=\lambda^{N} U\left(\lambda^{-N}\right)
$$


is convex on $(0, \infty)$. We further define $\mathcal{D} \mathcal{C}_{\infty}$ to be the set of such functions $U$ so that the function

$$
\psi(\lambda)=e^{\lambda} U\left(e^{-\lambda}\right)
$$

is convex on $(-\infty, \infty)$. A relevant example of an element of $\mathcal{D} \mathcal{C}_{N}$ is given by the function $U_{N}$ of $(3.5)$.

Definition 3.13 ([23, Definition 5.12]). Given $N \in[1, \infty]$, we say that a compact measured length space $(X, d, \nu)$ has nonnegative $N$-Ricci curvature if for all $\mu_{0}, \mu_{1} \in P(X)$ with $\operatorname{supp}\left(\mu_{0}\right) \subset \operatorname{supp}(\nu)$ and $\operatorname{supp}\left(\mu_{1}\right) \subset \operatorname{supp}(\nu)$, there is some Wasserstein geodesic $\left\{\mu_{t}\right\}_{t \in[0,1]}$ from $\mu_{0}$ to $\mu_{1}$ so that for all $U \in \mathcal{D} \mathcal{C}_{N}$ and all $t \in[0,1]$,

$$
U_{\nu}\left(\mu_{t}\right) \leq t U_{\nu}\left(\mu_{1}\right)+(1-t) U_{\nu}\left(\mu_{0}\right)
$$

We make some remarks about the definition.

REMARK 3.15. A similar definition in the case $N=\infty$, but in terms of $U=U_{\infty}$ instead of $U \in \mathcal{D} \mathcal{C}_{\infty}$, was used in [36, Definition 4.5]; see also Remark 5.5.

REMARK 3.16. It is not hard to show that if $(X, d, \nu)$ has nonnegative $N$-Ricci curvature and $N^{\prime} \geq N$ then $(X, d, \nu)$ has nonnegative $N^{\prime}$-Ricci curvature.

REMARK 3.17. Note that for $t \in(0,1)$, the intermediate measures $\mu_{t}$ are not required to have support in $\operatorname{supp}(\nu)$. If $(X, d, \nu)$ has nonnegative $N$-Ricci curvature then $\operatorname{supp}(\nu)$ is a convex subset of $X$ and $\left(\operatorname{supp}(\nu),\left.d\right|_{\operatorname{supp}(\nu)}, \nu\right)$ has nonnegative $N$-Ricci curvature [23, Theorem 5.53]. (We recall that a subset $A \subset X$ is convex if for any $x_{0}, x_{1} \in A$ there is a minimizing geodesic from $x_{0}$ to $x_{1}$ that lies entirely in $A$. It is totally convex if for any $x_{0}, x_{1} \in A$, any minimizing geodesic in $X$ from $x_{0}$ to $x_{1}$ lies in $A$.) So we don't lose much by assuming that $\operatorname{supp}(\nu)=X$.

REMARK 3.18. There is supposed to be a single Wasserstein geodesic $\left\{\mu_{t}\right\}_{t \in[0,1]}$ from $\mu_{0}$ to $\mu_{1}$ so that (3.14) holds along $\left\{\mu_{t}\right\}_{t \in[0,1]}$ for all $U \in \mathcal{D} \mathcal{C}_{N}$ simultaneously. However, (3.14) is only assumed to hold along some Wasserstein geodesic from $\mu_{0}$ to $\mu_{1}$, and not necessarily along all such Wasserstein geodesics. This is what we call weak displacement convexity. It may be more conventional to define convexity on a length space in terms of convexity along all geodesics. However, the definition with weak displacement convexity turns out to work better under MGH limits, and has most of the same implications as if we required convexity along all Wasserstein geodesics from $\mu_{0}$ to $\mu_{1}$. 
REMARK 3.19. Instead of requiring that (3.14) holds for all $U \in \mathcal{D} \mathcal{C}_{N}$, it would be consistent to make a definition in which it is only required to hold for the function $U=U_{N}$ of (3.5). For technical reasons, we prefer to require that (3.14) holds for all $U \in \mathcal{D} \mathcal{C}_{N}$; see Remark 6.11. Also, the class $\mathcal{D} \mathcal{C}_{N}$ is the natural class of functions for which the proof of Theorem 4.6 works.

3.2. MGH invariance. The next result says that Definition 3.13 satisfies Condition 1. of Wishlist 0.4. It shows that for each $N$, there is a self-contained world of measured length spaces with nonnegative $N$-Ricci curvature.

Theorem 3.20 ([23, Theorem 5.19], [36, Theorem 4.20], [37, Theorem 3.1]). Let $\left\{\left(X_{i}, d_{i}, \nu_{i}\right)\right\}_{i=1}^{\infty}$ be a sequence of compact measured length spaces with $\lim _{i \rightarrow \infty}\left(X_{i}, d_{i}, \nu_{i}\right)=(X, d, \nu)$ in the measured Gromov-Hausdorff topology. For any $N \in[1, \infty]$, if each $\left(X_{i}, d_{i}, \nu_{i}\right)$ has nonnegative $N$-Ricci curvature then $(X, d, \nu)$ has nonnegative $N$-Ricci curvature.

Proof. We give an outline of the proof. For simplicity, we just consider a single $U \in \mathcal{D} \mathcal{C}_{N}$; the same argument will allow one to handle all $U \in \mathcal{D} \mathcal{C}_{N}$ simultaneously.

Suppose first that $\mu_{0}$ and $\mu_{1}$ are absolutely continuous with respect to $\nu$, with continuous densities $\rho_{0}, \rho_{1} \in C(X)$. Let $\left\{f_{i}\right\}_{i=1}^{\infty}$ be a sequence of $\epsilon_{i}$-approximations as in Definition 0.2 . We first approximately-lift the measures $\mu_{0}$ and $\mu_{1}$ to $X_{i}$. That is, we use $f_{i}$ to pullback the densities to $X_{i}$, then multiply by $\nu_{i}$ and then normalize to get probability measures. More precisely, we put $\mu_{i, 0}=\frac{f_{i}^{*} \rho_{0} \nu_{i}}{\int_{X_{i}} f_{i}^{*} \rho_{0} d \nu_{i}} \in P\left(X_{i}\right)$ and $\mu_{i, 1}=\frac{f_{i}^{*} \rho_{1} \nu_{i}}{\int_{X_{i}} f_{i}^{*} \rho_{0} d \nu_{i}} \in P\left(X_{i}\right)$. One shows that $\lim _{i \rightarrow \infty}\left(f_{i}\right)_{*} \mu_{i, 0}=\mu_{0}$ and $\lim _{i \rightarrow \infty}\left(f_{i}\right)_{*} \mu_{i, 1}=\mu_{1}$ in the weak-* topology on $P(X)$. In addition, one shows that

$$
\lim _{i \rightarrow \infty} U_{\nu_{i}}\left(\mu_{i, 0}\right)=U_{\nu}\left(\mu_{0}\right)
$$

and

$$
\lim _{i \rightarrow \infty} U_{\nu_{i}}\left(\mu_{i, 1}\right)=U_{\nu}\left(\mu_{1}\right)
$$

Up on $X_{i}$, we are OK in the sense that by hypothesis, there is a Wasserstein geodesic $\left\{\mu_{i, t}\right\}_{t \in[0,1]}$ from $\mu_{i, 0}$ to $\mu_{i, 1}$ in $P\left(X_{i}\right)$ so that for all $t \in[0,1]$,

$$
U_{\nu_{i}}\left(\mu_{i, t}\right) \leq t U_{\nu_{i}}\left(\mu_{i, 1}\right)+(1-t) U_{\nu_{i}}\left(\mu_{i, 0}\right) .
$$

We now want to take a convergent subsequence of these Wasserstein geodesics in an appropriate sense to get a Wasserstein geodesic in $P(X)$. This can be done using Proposition 1.3 and an Arzela-Ascoli-type result. The conclusion is that after passing to a subsequence of the $i$ 's, there is a Wasserstein geodesic $\left\{\mu_{t}\right\}_{t \in[0,1]}$ from $\mu_{0}$ to $\mu_{1}$ in $P(X)$ so that for each $t \in[0,1]$, we have $\lim _{i \rightarrow \infty}\left(f_{i}\right)_{*} \mu_{i, t}=\mu_{t}$. 
Finally, we want to see what (3.23) becomes as $i \rightarrow \infty$. At the endpoints we have good limits from (3.21) and (3.22), so this handles the right-hand-side of (3.23) as $i \rightarrow \infty$. We do not have such a good limit for the left-hand-side. However, this is where the lower semicontinuity comes in. Applying parts (i) and (ii) of Proposition 3.8, we do know that

$$
U_{\nu}\left(\mu_{t}\right) \leq \liminf _{i \rightarrow \infty} U_{\left(f_{i}\right)_{*} \nu_{i}}\left(\left(f_{i}\right)_{*} \mu_{i, t}\right) \leq \liminf _{i \rightarrow \infty} U_{\nu_{i}}\left(\mu_{i, t}\right)
$$

This is enough to give the desired inequality (3.14) along the Wasserstein geodesic $\left\{\mu_{t}\right\}_{t \in[0,1]}$.

This handles the case when $\mu_{0}$ and $\mu_{1}$ have continuous densities. For general $\mu_{0}, \mu_{1} \in P(X)$, using mollifiers we can construct sequences $\left\{\mu_{j, 0}\right\}_{j=1}^{\infty}$ and $\left\{\mu_{j, 1}\right\}_{j=1}^{\infty}$ of absolutely continuous measures with continuous densities so that $\lim _{j \rightarrow \infty} \mu_{j, 0}=\mu_{0}$ and $\lim _{j \rightarrow \infty} \mu_{j, 1}=\mu_{1}$ in the weak-* topology. In addition, one can do the mollifying in such a way that $\lim _{j \rightarrow \infty} U_{\nu}\left(\mu_{j, 0}\right)=$ $U_{\nu}\left(\mu_{0}\right)$ and $\lim _{j \rightarrow \infty} U_{\nu}\left(\mu_{j, 1}\right)=U_{\nu}\left(\mu_{1}\right)$. ¿From what has already been shown, for each $j$ there is a Wasserstein geodesic $\left\{\mu_{j, t}\right\}_{t \in[0,1]}$ in $P(X)$ from $\mu_{j, 0}$ to $\mu_{j, 1}$ so that for all $t \in[0,1]$,

$$
U_{\nu}\left(\mu_{j, t}\right) \leq t U_{\nu}\left(\mu_{j, 1}\right)+(1-t) U_{\nu}\left(\mu_{j, 0}\right)
$$

After passing to a subsequence, we can assume that the Wasserstein geodesics $\left\{\mu_{j, t}\right\}_{t \in[0,1]}$ converge uniformly as $j \rightarrow \infty$ to a Wasserstein geodesic $\left\{\mu_{t}\right\}_{t \in[0,1]}$ from $\mu_{0}$ to $\mu_{1}$. From the lower semicontinuity of $U_{\nu}$, we have $U_{\nu}\left(\mu_{t}\right) \leq \liminf _{j \rightarrow \infty} U_{\nu}\left(\mu_{j, t}\right)$. Equation (3.14) follows.

3.3. Basic properties. We now give some basic properties of measured length spaces $(X, d, \nu)$ with nonnegative $N$-Ricci curvature.

Proposition 3.26 ([23, Proposition 5.20], [37, Theorem 2.3]). For $N \in$ $(1, \infty]$, if $(X, d, \nu)$ has nonnegative $N$-Ricci curvature then the measure $\nu$ is either a delta function or is nonatomic. The support of $\nu$ is a convex subset of $X$.

The next result is an analog of the Bishop-Gromov theorem.

Proposition 3.27 ([23, Proposition 5.27], [37, Theorem 2.3]). Suppose that $(X, d, \nu)$ has nonnegative $N$-Ricci curvature, with $N \in[1, \infty)$. Then for all $x \in \operatorname{supp}(\nu)$ and all $0<r_{1} \leq r_{2}$,

$$
\nu\left(B_{r_{2}}(x)\right) \leq\left(\frac{r_{2}}{r_{1}}\right)^{N} \nu\left(B_{r_{1}}(x)\right) .
$$

Proof. We give an outline of the proof. There is a Wasserstein geodesic $\left\{\mu_{t}\right\}_{t \in[0,1]}$ between $\mu_{0}=\delta_{x}$ and the restricted measure $\mu_{1}=\frac{1_{B_{r_{2}}(x)}}{\nu\left(B_{r_{2}}(x)\right)} \nu$, along which (3.14) holds. Such a Wasserstein geodesic comes from a fan of 
geodesics (the support of $\Pi$ ) that go from $x$ to points in $B_{r_{2}}(x)$. The actual transport, going backwards from $t=1$ to $t=0$, amounts to sliding the mass of $\mu_{1}$ along these geodesics towards $x$. In particular, the support of $\mu_{t}$ is contained in $B_{t r_{2}}(x)$. Applying (3.14) with $U=U_{N}$ and $t=\frac{r_{1}}{r_{2}}$, along with Holder's inequality, gives the desired result.

We give a technical result which will be used in deriving functional inequalities.

Proposition 3.29 ([23, Theorem 5.52]). Suppose that $(X, d, \nu)$ has nonnegative $N$-Ricci curvature. If $\mu_{0}$ and $\mu_{1}$ are absolutely continuous with respect to $\nu$ then the measures in the Wasserstein geodesic $\left\{\mu_{t}\right\}_{t \in[0,1]}$ of Definition 3.13 are all absolutely continuous with respect to $\nu$.

Finally, we mention that for nonbranching measured length spaces, there is a local-to-global principle which says that having nonnegative $N$-Ricci curvature in a local sense implies nonnegative $N$-Ricci curvature in a global sense [36, Theorem 4.17],[39]. We do not know if this holds in the branching case.

\section{Smooth metric-measure spaces}

We now address Condition 2. of Wishlist 0.4. We want to know what our abstract definition of "nonnegative $N$-Ricci curvature" boils down to in the classical Riemannian case. To be a bit more general, we allow Riemannian manifolds with weights. Let us say that a smooth measured length space consists of a smooth $n$-dimensional Riemannian manifold $M$ along with a smooth probability measure $\nu=e^{-\Psi} \operatorname{dvol}_{M}$. We write $(M, g, \nu)$ for the corresponding measured length space. We are taking $M$ to be compact.

Let us discuss possible Ricci tensors for smooth measured length spaces. If $\Psi$ is constant, i.e., if $\nu=\frac{\operatorname{dvol}_{M}}{\operatorname{vol}(M)}$, then the right notion of a Ricci tensor for $M$ is clearly just the usual Ric.

For general $\Psi$, a modified Ricci tensor

$$
\operatorname{Ric}_{\infty}=\operatorname{Ric}+\operatorname{Hess}(\Psi)
$$

was introduced by Bakry and Émery $[\mathbf{3}]$. (Note that the standard $\mathbb{R}^{n}$ with the Gaussian measure $(2 \pi)^{-\frac{n}{2}} e^{-\frac{|x|^{2}}{2}} d^{n} x$ has a constant Bakry-Émery tensor given by $\left(\operatorname{Ric}_{\infty}\right)_{i j}=\delta_{i j}$.) Their motivation came from a desire to generalize the Lichnerowicz inequality for the lower positive eigenvalue $\lambda_{1}(\triangle)$ of the Laplacian. We recall the Lichnerowicz result that if an $n$-dimensional Riemannian manifold has Ric $\geq K g$ with $K>0$ then $\lambda_{1}(\triangle) \geq \frac{n}{n-1} K[\mathbf{2 0}$.

In the case of a Riemannian manifold with a smooth probability measure $\nu=e^{-\Psi} \operatorname{dvol}_{M}$, there is a natural self-adjoint Laplacian $\widetilde{\triangle}$ acting on the 
weighted $L^{2}$-space $L^{2}\left(M, e^{-\Psi} \operatorname{dvol}_{M}\right)$, given by

$$
\int_{M} f_{1}\left(\widetilde{\triangle} f_{2}\right) e^{-\Psi} \operatorname{dvol}_{M}=\int_{M}\left\langle\nabla f_{1}, \nabla f_{2}\right\rangle e^{-\Psi} \operatorname{dvol}_{M}
$$

for $f_{1}, f_{2} \in C^{\infty}(M)$. Here $\left\langle\nabla f_{1}, \nabla f_{2}\right\rangle$ is the usual local inner product computed using the Riemannian metric $g$. Bakry and Émery showed that if $\operatorname{Ric}_{\infty} \geq K g$ then $\lambda_{1}(\widetilde{\triangle}) \geq K$. Although this statement is missing the $\frac{n}{n-1}$ factor of the Lichnerowicz inequality, it holds independently of $n$ and so can be considered to be a version of the Lichnerowicz inequality where one allows weights and takes $n \rightarrow \infty$. We refer to [1] for more information on the Bakry-Émery tensor Ric $_{\infty}$, including its relationship to log Sobolev inequalities. Some geometric properties of $\mathrm{Ric}_{\infty}$ were studied in [22]. More recently, the Bakry-Émery tensor has appeared as the right-hand-side of Perelman's modified Ricci flow equation [31].

We have seen that Ric $_{\infty}$ is a sort of Ricci tensor for the smooth measured length space $(M, g, \nu)$ when we consider $(M, g, \nu)$ to have "effective dimension" infinity. There is a similar tensor for other effective dimensions. Namely, if $N \in(n, \infty)$ then we put

$$
\operatorname{Ric}_{N}=\operatorname{Ric}+\operatorname{Hess}(\Psi)-\frac{1}{N-n} d \Psi \otimes d \Psi,
$$

where $\operatorname{dim}(M)=n$. The intuition is that $(M, g, \nu)$ has conventional dimension $n$ but is pretending to have dimension $N$, and $\operatorname{Ric}_{N}$ is its effective Ricci tensor under this pretence. There is now a sharp analog of the Lichnerowicz inequality: if $\operatorname{Ric}_{N} \geq K g$ with $K>0$ then $\lambda_{1}(\widetilde{\triangle}) \geq \frac{N}{N-1} K[\mathbf{2}]$. Geometric properties of $\operatorname{Ric}_{N}$ were studied in [22] and [32].

Finally, if $N<n$, or if $N=n$ and $\Psi$ is not locally constant, then we take the effective Ricci tensor $\operatorname{Ric}_{N}$ to be $-\infty$. To summarize,

Definition 4.4. For $N \in[1, \infty]$, define the $N$-Ricci tensor $\operatorname{Ric}_{N}$ of $(M, g, \nu)$ by

$$
\operatorname{Ric}_{N}= \begin{cases}\operatorname{Ric}+\operatorname{Hess}(\Psi) & \text { if } N=\infty, \\ \operatorname{Ric}+\operatorname{Hess}(\Psi)-\frac{1}{N-n} d \Psi \otimes d \Psi & \text { if } n<N<\infty, \\ \operatorname{Ric}+\operatorname{Hess}(\Psi)-\infty(d \Psi \otimes d \Psi) & \text { if } N=n, \\ -\infty & \text { if } N<n,\end{cases}
$$

where by convention $\infty \cdot 0=0$.

We can now state what the abstract notion of nonnegative $N$-Ricci curvature boils down to in the smooth case.

Theorem 4.6 ([23, Theorems 7.3 and 7.42], [36, Theorem 4.9], [37, Theorem 1.7]). Given $N \in[1, \infty]$, the measured length space $(M, g, \nu)$ has nonnegative $N$-Ricci curvature in the sense of Definition 3.13 if and only if $\operatorname{Ric}_{N} \geq 0$. 
The proof of Theorem 4.6 uses the explicit description of optimal transport on Riemannian manifolds.

In the special case when $\Psi$ is constant, and so $\nu=\frac{\operatorname{dvol}_{M}}{\operatorname{vol}(M)}$, Theorem 4.6 shows that we recover the usual notion of nonnegative Ricci curvature from our length space definition as soon as $N \geq n$.

4.1. Ricci limit spaces. We give an application of Theorems 3.20 and 4.6 to Ricci limit spaces. From Gromov precompactness, given $N \in \mathbb{Z}^{+}$ and $D>0$, the Riemannian manifolds with nonnegative Ricci curvature, dimension at most $N$ and diameter at most $D$ form a precompact subset of the set of measured length spaces, with respect to the MGH topology. The problem is to characterize the limit points. In general the limit points can be very singular, so this is a hard problem. However, let us ask a simpler question: what are the limit points that happen to be smooth measured length spaces? That is, we are trying to characterize the smooth limit points.

Corollary $4.7\left(\left[\mathbf{2 3}\right.\right.$, Corollary 7.45]). If $\left(B, g_{B}, e^{-\Psi} \mathrm{dvol}_{B}\right)$ is a measured Gromov-Hausdorff limit of Riemannian manifolds with nonnegative Ricci curvature and dimension at most $N$ then $\operatorname{Ric}_{N}(B) \geq 0$. (Here $B$ has dimension $n$, which is less than or equal to $N$.)

Proof. Suppose that $\left\{\left(M_{i}, g_{i}\right)\right\}_{i=1}^{\infty}$ is a sequence of Riemannian manifolds with nonnegative Ricci curvature and dimension at most $N$, with $\lim _{i \rightarrow \infty}\left(M_{i}, g_{i}, \frac{\operatorname{dvol}_{M_{i}}}{\operatorname{vol}\left(M_{i}\right)}\right)=\left(B, g_{B}, e^{-\Psi} \mathrm{dvol}_{B}\right)$. From Theorem 4.6, the measured length space $\left(M_{i}, g_{i}, \frac{\operatorname{dvol}_{M_{i}}}{\operatorname{vol}\left(M_{i}\right)}\right)$ has nonnegative $N$-Ricci curvature. From Theorem 3.20, $\left(B, g_{B}, e^{-\Psi} \mathrm{dvol}_{B}\right)$ has nonnegative $N$-Ricci curvature. From Theorem 4.6 again, $\operatorname{Ric}_{N}(B) \geq 0$.

There is a partial converse to Corollary 4.7 .

Proposition 4.8 ([23, Corollary 7.45]).

(i) Suppose that $N$ is an integer. If $\left(B, g_{B}, e^{-\Psi} \operatorname{dvol}_{B}\right)$ has $\operatorname{Ric}_{N}(B) \geq$ 0 with $N \geq \operatorname{dim}(B)+2$ then $\left(B, g_{B}, e^{-\Psi} \mathrm{dvol}_{B}\right)$ is a measured Gromov-Hausdorff limit of Riemannian manifolds with nonnegative Ricci curvature and dimension $N$.

(ii) Suppose that $N=\infty$. If $\left(B, g_{B}, e^{-\Psi} \operatorname{dvol}_{B}\right)$ has $\operatorname{Ric}_{\infty}(B) \geq 0$ then $\left(B, g_{B}, e^{-\Psi} \mathrm{dvol}_{B}\right)$ is a measured Gromov-Hausdorff limit of Riemannian manifolds $M_{i}$ with $\operatorname{Ric}\left(M_{i}\right) \geq-\frac{1}{i} g_{M_{i}}$.

ProOF. Let us consider part (i). The proof uses the warped product construction of [22]. Let $g_{S^{N-\operatorname{dim}(B)}}$ be the standard metric on the sphere $S^{N-\operatorname{dim}(B)}$. Let $M_{i}$ be $B \times S^{N-\operatorname{dim}(B)}$ with the warped product metric

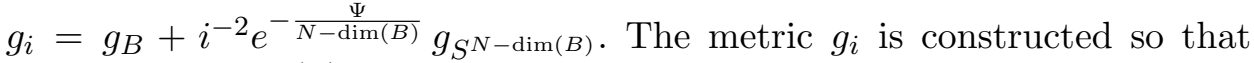
if $p: B \times S^{N-\operatorname{dim}(B)} \rightarrow B$ is projection onto the first factor then $p_{*} \mathrm{dvol}_{M_{i}}$ 
is a constant times $e^{-\Psi} \operatorname{dvol}_{B}$. In terms of the fibration $p$, the Ricci tensor of $M_{i}$ splits into horizontal and vertical components, with the horizontal component being exactly $\operatorname{Ric}_{N}$. As $i$ increases, the fibers shrink and the vertical Ricci curvature of $M_{i}$ becomes dominated by the Ricci curvature of the small fiber $S^{N-\operatorname{dim}(B)}$, which is positive as we are assuming that $N-\operatorname{dim}(B) \geq 2$. Then for large $i,\left(M_{i}, g_{i}\right)$ has nonnegative Ricci curvature. Taking $f_{i}=p$, we see that $\lim _{i \rightarrow \infty}\left(M_{i}, g_{i}, \frac{\operatorname{dvol}_{M_{i}}}{\operatorname{vol}\left(M_{i}\right)}\right)=\left(B, g_{B}, e^{-\Psi} \mathrm{dvol}_{B}\right)$.

The proof of (ii) is similar, except that we also allow the dimensions of the fibers to go to infinity.

Examples of singular spaces with nonnegative $N$-Ricci curvature come from group actions. Suppose that a compact Lie group $G$ acts isometrically on a $N$-dimensional Riemannian manifold $M$ that has nonnegative Ricci curvature. Put $X=M / G$, let $p: M \rightarrow X$ be the quotient map, let $d$ be the quotient metric and put $\nu=p_{*}\left(\frac{\operatorname{dvol}_{M}}{\operatorname{vol}(M)}\right)$. Then $(X, d, \nu)$ has nonnegative $N$-Ricci curvature [23, Corollary 7.51].

Finally, we recall the theorem of O'Neill that sectional curvature is nondecreasing under pushforward by a Riemannian submersion. There is a Ricci analog of the O'Neill theorem, expressed in terms of the modified Ricci tensor $\operatorname{Ric}_{N}[\mathbf{2 2}]$. The proof of this in [22] was by explicit tensor calculations. Using optimal transport, one can give a "synthetic" proof of this Ricci O'Neill theorem [23, Corollary 7.52]. (This is what first convinced the author that optimal transport is the right approach.)

REMARK 4.9. We return to the question of whether one can give a good definition of "nonnegative $N$-Ricci curvature" by just taking the conclusion of the Bishop-Gromov theorem and turning it into a definition. To be a bit more reasonable, we consider taking an angular Bishop-Gromov inequality as the definition. Such an inequality, with parameter $n$, does indeed characterize when an $n$-dimensional Riemannian manifold has nonnegative Ricci curvature. Namely, from comparison geometry, nonnegative Ricci curvature implies an angular Bishop-Gromov inequality. To go the other way, suppose that the angular Bishop-Gromov inequality holds. We use polar coordinates around a point $m \in M$ and recall that the volume of a infinitesimally small angular sector centered in the direction of a unit vector $v \in T_{m} M$, and going up to radius $r$, has the Taylor expansion

$$
V(v, r)=\text { const. } r^{n}\left(1-\frac{n}{6(n+2)} \operatorname{Ric}(v, v) r^{2}+\cdots\right) .
$$

If $r^{-n} V(v, r)$ is to be nonincreasing in $r$ then we must have $\operatorname{Ric}(v, v) \geq 0$. As $m$ and $v$ were arbitrary, we conclude that Ric $\geq 0$.

There is a version of the angular Bishop-Gromov inequality for measured length spaces, called the "measure contracting property" (MCP) [28, 37]. It satisfies Condition 1 of Wishlist 0.4. 
The reason that the MCP notion is not entirely satisfactory can be seen by asking what it takes for a smooth measured length space $\left(M, g, e^{-\Psi} \mathrm{dvol}_{M}\right)$ to satisfy the $N$-dimensional angular Bishop-Gromov inequality. (Here $\operatorname{dim}(M)=n$.) There is a Riccati-type inequality

$$
\frac{\partial}{\partial r}\left(\operatorname{Tr} \Pi-\frac{\partial \Psi}{\partial r}\right) \leq-\operatorname{Ric}_{N}\left(\partial_{r}, \partial_{r}\right)-\frac{1}{N-1}\left(\operatorname{Tr} \Pi-\frac{\partial \Psi}{\partial r}\right)^{2},
$$

which looks good. Again there is an expansion for the measure of the infinitesimally small angular sector considered above, of the form $\widehat{V}(r)=r^{n}\left(a_{0}+\right.$ $\left.a_{1} r+a_{2} r^{2}+\cdots\right)$, where the coefficents $a_{i}$ can be expressed in terms of curvature derivatives and the derivatives of $\Psi$. However, if $N>n$ then saying that $r^{-N} \widehat{V}(r)$ is nonincreasing in $r$ does not imply anything about the coefficients. Thus having the $N$-dimensional angular Bishop-Gromov inequality does not imply that $\operatorname{Ric}_{N} \geq 0$. In particular, it does not seem that one can prove Corollary 4.7 using MCP.

Having nonnegative $N$-Ricci curvature does imply MCP [37].

\section{5. $N$-Ricci curvature bounded below by $K$}

In Section 3 we gave the definition of nonnegative $N$-Ricci curvature. In this section we discuss how to extend this to a notion of a measured length space having $N$-Ricci curvature bounded below by some real number $K$.

We start with the case $N=\infty$. As mentioned in Section 2, formal computations indicate that in the case of a smooth measured length space $\left(M, g, e^{-\Psi} \operatorname{dvol}_{M}\right)$, having $\operatorname{Ric}_{\infty} \geq K g$ should imply that $H_{\infty}$ has Hessian bounded below by $K g_{H^{-1}}$ on $P(M)$. In particular, if $\left\{\mu_{t}\right\}_{t \in[0,1]}$ is a geodesic in $P(M)$ then we would expect that $H_{\infty}\left(\mu_{t}\right)-\frac{K}{2} W_{2}\left(\mu_{0}, \mu_{1}\right)^{2} t^{2}$ is convex in $t$. This motivates an adaption of Definition 3.13.

In order to handle all $U \in \mathcal{D C}_{\infty}$, we first make the following definition. Given a continuous convex function $U:[0, \infty) \rightarrow \mathbb{R}$, we define its "pressure" by

$$
p(r)=r U_{+}^{\prime}(r)-U(r),
$$

where $U_{+}^{\prime}(r)$ is the right-derivative. Then given $K \in \mathbb{R}$, we define $\lambda: \mathcal{D} \mathcal{C}_{\infty} \rightarrow$ $\mathbb{R} \cup\{-\infty\}$ by

$$
\lambda(U)=\inf _{r>0} K \frac{p(r)}{r}= \begin{cases}K \lim _{r \rightarrow 0^{+}} \frac{p(r)}{r} & \text { if } K>0, \\ 0 & \text { if } K=0, \\ K \lim _{r \rightarrow \infty} \frac{p(r)}{r} & \text { if } K<0 .\end{cases}
$$

Note that if $U=U_{\infty}$ (recall that $U_{\infty}(r)=r \log r$ ) then $p(r)=r$ and so $\lambda\left(U_{\infty}\right)=K$. 
Definition 5.3 ([23, Definition 5.13]). Given $K \in \mathbb{R}$, we say that $(X, d, \nu)$ has $\infty$-Ricci curvature bounded below by $K$ if for all $\mu_{0}, \mu_{1} \in P(X)$ with $\operatorname{supp}\left(\mu_{0}\right) \subset \operatorname{supp}(\nu)$ and $\operatorname{supp}\left(\mu_{1}\right) \subset \operatorname{supp}(\nu)$, there is some Wasserstein geodesic $\left\{\mu_{t}\right\}_{t \in[0,1]}$ from $\mu_{0}$ to $\mu_{1}$ so that for all $U \in \mathcal{D C}_{\infty}$ and all $t \in[0,1]$

$$
U_{\nu}\left(\mu_{t}\right) \leq t U_{\nu}\left(\mu_{1}\right)+(1-t) U_{\nu}\left(\mu_{0}\right)-\frac{1}{2} \lambda(U) t(1-t) W_{2}\left(\mu_{0}, \mu_{1}\right)^{2} .
$$

REMARK 5.5. A similar definition, but in terms of $U=U_{\infty}$ instead of $U \in \mathcal{D C}_{\infty}$, was used in [36, Definition 4.5].

Clearly if $K=0$ then we recover the notion of nonnegative $\infty$-Ricci curvature in the sense of Definition 3.13. The $N=\infty$ results of Sections 3 and 4 can be extended to the present case where $K$ may be nonzero.

A good notion of $(X, d, \nu)$ having $N$-Ricci curvature bounded below by $K \in \mathbb{R}$, where $N$ can be finite, is less clear and is essentially due to Sturm [37]. The following definition is a variation of Sturm's definition and appears in $[24]$.

Given $K \in \mathbb{R}$ and $N \in(1, \infty]$, define

$$
\beta_{t}\left(x_{0}, x_{1}\right)= \begin{cases}e^{\frac{1}{6} K\left(1-t^{2}\right) d\left(x_{0}, x_{1}\right)^{2}} & \text { if } N=\infty, \\ \infty & \text { if } N<\infty, K>0 \text { and } \alpha>\pi, \\ \left(\frac{\sin (t \alpha)}{t \sin \alpha}\right)^{N-1} & \text { if } N<\infty, K>0 \text { and } \alpha \in[0, \pi], \\ 1 & \text { if } N<\infty \text { and } K=0, \\ \left(\frac{\sinh (t \alpha)}{t \sinh \alpha}\right)^{N-1} & \text { if } N<\infty \text { and } K<0,\end{cases}
$$

where

$$
\alpha=\sqrt{\frac{|K|}{N-1}} d\left(x_{0}, x_{1}\right)
$$

When $N=1$, define

$$
\beta_{t}\left(x_{0}, x_{1}\right)= \begin{cases}\infty & \text { if } K>0 \\ 1 & \text { if } K \leq 0\end{cases}
$$

Although we may not write it explicitly, $\alpha$ and $\beta$ depend on $K$ and $N$.

We can disintegrate a transference plan $\pi$ with respect to its first marginal $\mu_{0}$ or its second marginal $\mu_{1}$. We write this in a slightly informal way:

$$
d \pi\left(x_{0}, x_{1}\right)=d \pi\left(x_{1} \mid x_{0}\right) d \mu_{0}\left(x_{0}\right)=d \pi\left(x_{0} \mid x_{1}\right) d \mu_{1}\left(x_{1}\right) .
$$

Definition 5.10. [24] We say that $(X, d, \nu)$ has $N$-Ricci curvature bounded below by $K$ if the following condition is satisfied. Given $\mu_{0}, \mu_{1} \in$ 
$P(X)$ with support in $\operatorname{supp}(\nu)$, write their Lebesgue decompositions with respect to $\nu$ as $\mu_{0}=\rho_{0} \nu+\mu_{0, s}$ and $\mu_{1}=\rho_{1} \nu+\mu_{1, s}$, respectively. Then there is some optimal dynamical transference plan $\Pi$ from $\mu_{0}$ to $\mu_{1}$, with corresponding Wasserstein geodesic $\mu_{t}=\left(e_{t}\right)_{*} \Pi$, so that for all $U \in \mathcal{D C}_{N}$ and all $t \in[0,1]$, we have

$$
\begin{aligned}
U_{\nu}\left(\mu_{t}\right) \leq(1 & -t) \int_{X \times X} \beta_{1-t}\left(x_{0}, x_{1}\right) U\left(\frac{\rho_{0}\left(x_{0}\right)}{\beta_{1-t}\left(x_{0}, x_{1}\right)}\right) d \pi\left(x_{1} \mid x_{0}\right) d \nu\left(x_{0}\right) \\
& +t \int_{X \times X} \beta_{t}\left(x_{0}, x_{1}\right) U\left(\frac{\rho_{1}\left(x_{1}\right)}{\beta_{t}\left(x_{0}, x_{1}\right)}\right) d \pi\left(x_{0} \mid x_{1}\right) d \nu\left(x_{1}\right) \\
& +U^{\prime}(\infty)\left[(1-t) \mu_{0, s}[X]+t \mu_{1, s}[X]\right] .
\end{aligned}
$$

Here if $\beta_{t}\left(x_{0}, x_{1}\right)=\infty$ then we interpret $\beta_{t}\left(x_{0}, x_{1}\right) U\left(\frac{\rho_{1}\left(x_{1}\right)}{\beta_{t}\left(x_{0}, x_{1}\right)}\right)$ as $U^{\prime}(0) \rho_{1}\left(x_{1}\right)$, and similarly for $\beta_{1-t}\left(x_{0}, x_{1}\right) U\left(\frac{\rho_{0}\left(x_{0}\right)}{\beta_{1-t}\left(x_{0}, x_{1}\right)}\right)$.

REMARK 5.12. If $\mu_{0}$ and $\mu_{1}$ are absolutely continuous with respect to $\nu$ then the inequality can be rewritten in the more symmetric form

$$
\begin{gathered}
U_{\nu}\left(\mu_{t}\right) \leq(1-t) \int_{X \times X} \frac{\beta_{1-t}\left(x_{0}, x_{1}\right)}{\rho_{0}\left(x_{0}\right)} U\left(\frac{\rho_{0}\left(x_{0}\right)}{\beta_{1-t}\left(x_{0}, x_{1}\right)}\right) d \pi\left(x_{0}, x_{1}\right) \\
+t \int_{X \times X} \frac{\beta_{t}\left(x_{0}, x_{1}\right)}{\rho_{1}\left(x_{1}\right)} U\left(\frac{\rho_{1}\left(x_{1}\right)}{\beta_{t}\left(x_{0}, x_{1}\right)}\right) d \pi\left(x_{0}, x_{1}\right) .
\end{gathered}
$$

Remark 5.14. Given $K \geq K^{\prime}$ and $N \leq N^{\prime}$, if $(X, d, \nu)$ has $N$-Ricci curvature bounded below by $K$ then it also has $N^{\prime}$-Ricci curvature bounded below by $K^{\prime}$.

REMARK 5.15. The case $N=\infty$ of Definition 5.10 is not quite the same as what we gave in Definition 5.3! However, it is true that having $\infty$-Ricci curvature bounded below by $K$ in the sense of Definition 5.10 implies that one has $\infty$-Ricci curvature bounded below by $K$ in the sense of Definition 5.3 $[24]$. Hence any $N=\infty$ consequences of Definition 5.3 are also consequences of Definition 5.10. We include the $N=\infty$ case in Definition 5.10 in order to present a unified treatment, but this example shows that there may be some flexibility in the precise definitions.

The results of Sections 3 and 4 now have extensions to the case $K \neq 0$. However, the proofs of some of the extensions, such as that of Theorem 3.20, may become much more involved [37, Theorem 3.1], [39].

Using the extension of Proposition 3.27, one obtains a generalized Bonnet-Myers theorem.

Proposition 5.16 ([37, Corollary 2.6]). If $(X, d, \nu)$ has $N$-Ricci curvature bounded below by $K>0$ then $\operatorname{supp}(\nu)$ has diameter bounded above by $\sqrt{\frac{N-1}{K}} \pi$. 


\section{Analytic consequences}

Lower Ricci curvature bounds on Riemannian manifolds have various analytic implications, such as eigenvalue inequalities, Sobolev inequalities and local Poincaré inequalities. It turns out that these inequalities pass to our generalized setting.

6.1. Log Sobolev and Poincaré inequalities. Let us first discuss the so-called log Sobolev inequality. If a smooth measured length space $\left(M, g, e^{-\Psi} \operatorname{dvol}_{M}\right)$ has $\operatorname{Ric}_{\infty} \geq K g$, with $K>0$, then for all $f \in C^{\infty}(M)$ with $\int_{M} f^{2} e^{-\Psi} \operatorname{dvol}_{M}=1$, it was shown in $[\mathbf{3}]$ that

$$
\int_{M} f^{2} \log \left(f^{2}\right) e^{-\Psi} \operatorname{dvol}_{M} \leq \frac{2}{K} \int_{M}|\nabla f|^{2} e^{-\Psi} \operatorname{dvol}_{M} .
$$

The standard log Sobolev inequality on $\mathbb{R}^{n}$ comes from taking

$$
d \nu=(4 \pi)^{-\frac{n}{2}} e^{-|x|^{2}} d^{n} x
$$

giving

$$
\int_{\mathbb{R}^{n}} f^{2} \log \left(f^{2}\right) e^{-|x|^{2}} d^{n} x \leq \int_{\mathbb{R}^{n}}|\nabla f|^{2} e^{-|x|^{2}} d^{n} x
$$

whenever $(4 \pi)^{-\frac{n}{2}} \int_{\mathbb{R}^{n}} f^{2} e^{-|x|^{2}} d^{n} x=1$.

The $\log$ Sobolev inequality for $\left(M, g, e^{-\Psi} \operatorname{dvol}_{M}\right)$ was given both heuristic and rigorous optimal transport proofs by Otto and Villani [30]. We describe the heuristic proof here. From Section 2, having $\mathrm{Ric}_{\infty} \geq K g$ formally implies that Hess $\left(H_{\infty}\right) \geq K g_{H^{-1}}$ on $P(M)$. Take $\mu_{0}=e^{-\Psi} \operatorname{dvol}_{M}$ and $\mu_{1}=f^{2} e^{-\Psi} \operatorname{dvol}_{M}$. Let $\left\{\mu_{t}\right\}_{t \in[0,1]}$ be a Wasserstein geodesic from $\mu_{0}$ to $\mu_{1}$ along which

$$
F(t)=H_{\infty}\left(\mu_{t}\right)-\frac{K}{2} W_{2}\left(\mu_{0}, \mu_{1}\right)^{2} t^{2}
$$

is convex in $t$. As $F(0)=0$, we have $F(1) \leq F^{\prime}(1)$, or

$$
\begin{aligned}
& H_{\infty}\left(\mu_{1}\right)-\frac{K}{2} W_{2}\left(\mu_{0}, \mu_{1}\right)^{2} \\
& \leq\left\langle\left.\frac{d \mu_{t}}{d t}\right|_{t=1},\left(\operatorname{grad} H_{\infty}\right)\left(\mu_{1}\right)\right\rangle_{g_{H^{-1}}}-K W_{2}\left(\mu_{0}, \mu_{1}\right)^{2} \\
& \leq\left|\frac{d \mu_{t}}{d t}\right|_{t=1}|\cdot|\left(\operatorname{grad} H_{\infty}\right)\left(\mu_{1}\right) \mid-K W_{2}\left(\mu_{0}, \mu_{1}\right)^{2} .
\end{aligned}
$$

Here $\operatorname{grad} H_{\infty}$ is the formal gradient of $H_{\infty}$ on $P(M)$ and the last norms denote lengths with respect to $g_{H^{-1}}$. As $\left\{\mu_{t}\right\}_{t \in[0,1]}$ is a minimizing geodesic 
from $\mu_{0}$ to $\mu_{1}$, we should have

$$
\left|\frac{d \mu_{t}}{d t}\right|_{t=1} \mid=W_{2}\left(\mu_{0}, \mu_{1}\right) .
$$

A formal computation gives

$$
\left|\left(\operatorname{grad} H_{\infty}\right)\left(\mu_{1}\right)\right|^{2}=4 \int_{M}|\nabla f|^{2} e^{-\Psi} \operatorname{dvol}_{M} .
$$

Then

$$
\begin{aligned}
& \int_{M} f^{2} \log \left(f^{2}\right) e^{-\Psi} \mathrm{dvol}_{M} \\
& \leq 2 W_{2}\left(\mu_{0}, \mu_{1}\right) \sqrt{\int_{M}|\nabla f|^{2} e^{-\Psi} \operatorname{dvol}_{M}}-\frac{K}{2} W_{2}\left(\mu_{0}, \mu_{1}\right)^{2} \\
& \leq \sup _{w \in \mathbb{R}}\left(2 w \sqrt{\int_{M}|\nabla f|^{2} e^{-\Psi} \operatorname{dvol}_{M}}-\frac{K}{2} w^{2}\right) \\
& =\frac{2}{K} \int_{M}|\nabla f|^{2} e^{-\Psi} \operatorname{dvol}_{M}
\end{aligned}
$$

which is the log Sobolev inequality.

The rigorous optimal transport proof in $[\mathbf{3 0}]$ extends to measured length spaces. To give the statement, we first must say what we mean by $|\nabla f|$. We define the local gradient norm of a Lipschitz function $f \in \operatorname{Lip}(X)$ by the formula

$$
|\nabla f|(x)=\limsup _{y \rightarrow x} \frac{|f(y)-f(x)|}{d(x, y)} .
$$

We don't claim to know the meaning of the gradient $\nabla f$ on $X$ in this generality, but we can talk about its norm anyway! Then we have the following log Sobolev inequality for measured length spaces.

TheOREM 6.9 ([23, Corollary 6.12]). Suppose that a compact measured length space $(X, d, \nu)$ has $\infty$-Ricci curvature bounded below by $K>0$, in the sense of Definition 5.3. Suppose that $f \in \operatorname{Lip}(X)$ satisfies $\int_{X} f^{2} d \nu=1$. Then

$$
\int_{X} f^{2} \log \left(f^{2}\right) d \nu \leq \frac{2}{K} \int_{X}|\nabla f|^{2} d \nu .
$$

In the case of Riemannian manifolds, one recovers from (6.10) the log Sobolev inequality (6.1) of Bakry and Émery.

REMARK 6.11. The proof of Theorem 6.9, along with the other inequalities in this section, uses the $K>0$ analog of Proposition 3.29. In turn, the proof of Proposition 3.29 uses the fact that (3.14) holds for all $U \in \mathcal{D C}_{N}$, as opposed to just $U_{N}$. 
As is well-known, one can obtain a Poincaré inequality from (6.10). Take $h \in \operatorname{Lip}(X)$ with $\int_{X} h d \nu=0$ and put $f^{2}=1+\epsilon h$. Taking $\epsilon$ small and expanding the two sides of (6.10) in $\epsilon$ gives the following result.

Corollary 6.12 ([23, Theorem 6.18]). Suppose that a compact measured length space $(X, d, \nu)$ has $\infty$-Ricci curvature bounded below by $K>0$. Then for all $h \in \operatorname{Lip}(X)$ with $\int_{X} h d \nu=0$, we have

$$
\int_{X} h^{2} d \nu \leq \frac{1}{K} \int_{X}|\nabla h|^{2} d \nu
$$

In case of a smooth measured length space $\left(M, g, e^{-\Psi} \mathrm{dvol}_{M}\right)$, the inequality (6.13) coincides with the Bakry-Émery extension of the Lichnerowicz inequality, namely $\lambda_{1}(\widetilde{\triangle}) \geq K$. For a general measured length space as in the hypotheses of Corollary 6.12, we do not know if there is a well-defined Laplacian. The Poincaré inequality of Corollary 6.12 can be seen as a generalized eigenvalue inequality that avoids this issue. To say a bit more about when one does have a Laplacian, if $Q(h)=\int_{X}|\nabla h|^{2} d \nu$ defines a quadratic form on $\operatorname{Lip}(X)$, which in addition is closable in $L^{2}(X, \nu)$, then there is a self-adjoint Laplacian $\triangle_{\nu}$ associated to $Q$. In this case, Corollary 6.12 implies that $\triangle_{\nu} \geq K$ on the orthogonal complement of the constant functions.

In the case of a Ricci limit space, Cheeger and Colding used additional structure in order to show the Laplacian does exist [10].

6.2. Sobolev inequality. The log Sobolev inequality can be viewed as an infinite-dimensional version of an ordinary Sobolev inequality. As such, it is interesting because it is a dimension-independent result. However, if one has $N$-Ricci curvature bounded below by $K>0$ with $N$ finite then one gets an ordinary Sobolev inequality, which is a sharper result.

Proposition 6.14 ([24]). Given $N \in(1, \infty)$ and $K>0$, suppose that $(X, d, \nu)$ has $N$-Ricci curvature bounded below by $K$. Then for any nonnegative Lipschitz function $\rho_{0} \in \operatorname{Lip}(X)$ with $\int_{X} \rho_{0} d \nu=1$, one has

$$
N-N \int_{X} \rho_{0}^{1-\frac{1}{N}} d \nu \leq \frac{1}{2 K}\left(\frac{N-1}{N}\right)^{2} \int_{X} \frac{\rho_{0}^{-1-\frac{2}{N}}}{\frac{1}{3}+\frac{2}{3} \rho_{0}^{-\frac{1}{N}}}\left|\nabla \rho_{0}\right|^{2} d \nu .
$$

To put Proposition 6.14 into a more conventional form, we give a slightly weaker inequality.

Proposition 6.16 ([24]). Given $N \in(2, \infty)$ and $K>0$, suppose that $(X, d, \nu)$ has $N$-Ricci curvature bounded below by $K$. Then for any nonnegative Lipschitz function $f \in \operatorname{Lip}(X)$ with $\int_{X} f^{\frac{2 N}{N-2}} d \nu=1$, one has

$$
1-\left(\int_{X} f d \nu\right)^{\frac{2}{N+2}} \leq \frac{6}{K N}\left(\frac{N-1}{N-2}\right)^{2} \int_{X}|\nabla f|^{2} d \nu .
$$


Putting (6.17) into a homogeneous form, the content of Proposition 6.16 is that there is a bound of the form $\|f\|_{\frac{2 N}{N-2}} \leq F\left(\|f\|_{1},\|\nabla f\|_{2}\right)$ for some appropriate function $F$. This is an example of Sobolev embedding. The inequality (6.17) is not sharp, due to the many approximations made in its derivation.

One can use Proposition 6.14 to prove a sharp Poincaré inequality.

Proposition $6.18([\mathbf{2 4}])$. Given $N \in(1, \infty)$ and $K>0$, suppose that $(X, d, \nu)$ has $N$-Ricci curvature bounded below by $K$. Suppose that $h \in$ $\operatorname{Lip}(X)$ has $\int_{X} h d \nu=0$. Then

$$
\int_{X} h^{2} d \nu \leq \frac{N-1}{K N} \int_{X}|\nabla h|^{2} d \nu
$$

In the case of an $N$-dimensional Riemannian manifold with Ric $\geq K g$, one recovers the Lichnerowicz inequality for the lowest positive eigenvalue of the Laplacian [20]. It is sharp on round spheres.

6.3. Local Poincaré inequality. When doing analysis on metricmeasure spaces, a useful analytic property is a "local" Poincaré inequality. A metric-measure space $(X, d, \nu)$ admits a local Poincaré inequality if, roughly speaking, for each function $f$ and each ball $B$ in $X$, the mean deviation (on $B$ ) of $f$ from its average value on $B$ is quantitatively controlled by the gradient of $f$ on a larger ball.

To make this precise, if $B=B_{r}(x)$ is a ball in $X$ then we write $\lambda B$ for $B_{\lambda r}(x)$. The measure $\nu$ is said to be doubling if there is some $D>0$ so that for all balls $B, \nu(2 B) \leq D \nu(B)$. An upper gradient for a function $u \in C(X)$ is a Borel function $g: X \rightarrow[0, \infty]$ such that for each curve $\gamma:[0,1] \rightarrow X$ with finite length $L(\gamma)$ and constant speed,

$$
|u(\gamma(1))-u(\gamma(0))| \leq L(\gamma) \int_{0}^{1} g(\gamma(t)) d t .
$$

If $u$ is Lipschitz then $|\nabla u|$ is an example of an upper gradient.

There are many forms of local Poincaré inequalities. The strongest one, in a certain sense, is as follows:

Definition 6.21. A metric-measure space $(X, d, \nu)$ admits a local Poincaré inequality if there are constants $\lambda \geq 1$ and $P<\infty$ such that for all $u \in C(X)$ and $B=B_{r}(x)$ with $\nu(B)>0$, each upper gradient $g$ of $u$ satisfies

$$
f_{B}\left|u-\langle u\rangle_{B}\right| d \nu \leq \operatorname{Pr} f_{\lambda B} g d \nu .
$$

Here the barred integral is the average (with respect to $\nu$ ), e.g., $f_{\lambda B} g d \nu=$ $\frac{\int_{\lambda B} g d \nu}{\nu(\lambda B)}$, and $\langle u\rangle_{B}$ is the average of $u$ over the ball $B$. In the case of a length 
space, the local Poincaré inequality as formulated in Definition 6.21 actually implies stronger inequalities, for which we refer to [15, Chapters 4 and 9]. It is known that the property of admitting a local Poincaré inequality is preserved under measured Gromov-Hausdorff limits [18, 19]. (This was also shown by Cheeger in unpublished work.) Cheeger showed that if a metric-measure space has a doubling measure and admits a local Poincaré inequality then it has remarkable extra local structure [6].

Cheeger and Colding showed that local Poincaré inequalities exist for Ricci limit spaces [10]. The method of proof was to show that Riemannian manifolds with lower Ricci curvature bounds satisfy a certain "segment inequality" [7, Theorem 2.11] and then to show that the property of satisfying the segment inequality is preserved under measured Gromov-Hausdorff limits [10, Theorem 2.6]. The segment inequality then implies the local Poincaré inequality.

It turns out that the argument using the segment inequality can be abstracted and applied to certain measured length spaces. For simplicity, we restrict to the case of nonnegative $N$-Ricci curvature. We say that $(X, d, \nu)$ has almost-everywhere unique geodesics if for $\nu \otimes \nu$-almost all $\left(x_{0}, x_{1}\right) \in$ $X \times X$, there is a unique minimizing geodesic $\gamma \in \Gamma$ with $\gamma(0)=x_{0}$ and $\gamma(1)=x_{1}$.

THEOREM $6.23([\mathbf{2 4}, \mathbf{3 4}, \mathbf{3 7}])$. If a compact measured length space $(X, d, \nu)$ has nonnegative $N$-Ricci curvature and almost-everywhere unique geodesics then it satisfies the local Poincaré inequality of Definition 6.21 with $\lambda=2$ and $P=2^{2 N+1}$.

As is well-known, a Riemannian manifold has almost-everywhere unique geodesics. A sufficient condition for $(X, d, \nu)$ to have almost-everywhere unique geodesics is that almost every $x \in X$ is nonbranching in a certain sense $[\mathbf{3 4}, \mathbf{3 7}]$.

The result of Theorem 6.23 holds in greater generality. What one needs is a way of joining up points by geodesics, called a "democratic coupling" in $[\mathbf{2 4}]$, and a doubling condition on the measure.

We do not know whether the condition of nonnegative $N$-Ricci curvature is sufficient in itself to imply a local Poincaré inequality. Having nonnegative $N$-Ricci curvature does not imply almost-everywhere unique geodesics. For a noncompact example, the finite-dimensional Banach space $\mathbb{R}^{n}$ with the $l_{1}$ norm and the Lebesgue measure has nonnegative $n$-Ricci curvature, but certainly does not have almost-everywhere unique geodesics.

\section{Final remarks}

In this survey we have concentrated on compact spaces. There is also a notion of Ricci curvature bounded below for noncompact measured length spaces $(X, d, \nu)[\mathbf{2 3}$, Appendix E]. Here we want $X$ to be a complete pointed locally compact length space and $\nu$ to be a nonnegative nonzero Radon 
measure on $X$. We do not require $\nu$ to be a probability measure. There is a Wasserstein space $P_{2}(X)$ of probability measures on $X$ with finite second moment, i.e.,

$$
P_{2}(X)=\left\{\mu \in P(X): \int_{X} d(\star, x)^{2} d \mu(x)<\infty\right\},
$$

where $\star$ is the basepoint in $X$. Many of the results described in this survey extend from compact spaces to such noncompact spaces, although interesting technical points arise.

In particular, if $(X, d, \nu)$ is a compact or noncompact space with nonnegative $N$-Ricci curvature and $\operatorname{supp}(\nu)=X$, and if $x$ is a point in $X$, then a tangent cone at $x$ has nonnegative $N$-Ricci curvature [23, Corollary E.44].

There are many directions for future research. Any specific problems that we write here may become obsolete, but let us just mention two general directions. One direction is to see whether known results about Riemannian manifolds with lower Ricci curvature bounds extend to measured length spaces with lower Ricci curvature bounds. As a caution, this is not always the case. For example, the Cheeger-Gromoll splitting theorem says that if there is a line in a complete Riemannian manifold $M$ with nonnegative Ricci curvature then there is an isometric splitting $M=\mathbb{R} \times Y$. This is not true for measured length spaces with nonnegative $N$-Ricci curvature. Counterexamples are given by nonEuclidean $n$-dimensional normed linear spaces, equipped with Lebesgue measure, which all have nonnegative $n$ Ricci curvature [39]. However, it is possible that there is some vestige of the splitting theorem left.

The splitting theorem does hold for a pointed Gromov-Hausdorff limit of a sequence $\left\{\left(M_{i}, g_{i}\right)\right\}_{i=1}^{\infty}$ of complete Riemannian manifolds with Ricci curvature bounded below by $-\frac{1}{i}[\mathbf{7}]$, so not every finite-dimensional $(X, d, \nu)$ with nonnegative $N$-Ricci curvature arises as a limit in this way. (The analogous statement is not known for finite-dimensional Alexandrov spaces, but there are candidate Alexandrov spaces that may not be Gromov-Hausdorff limits of Riemannian manifolds with sectional curvature uniformly bounded below [17].) One's attitude towards this fact may depend on whether one intuitively feels that finite-dimensional normed linear spaces should or should not have nonnegative Ricci curvature.

Another direction of research is to find classes of measured length spaces $(X, d, \nu)$ which do or do not have lower Ricci curvature bounds. This usually amounts to understanding optimal transport on such spaces.

\section{References}

[1] C. Ané, S. Blachère, D. Chafaï, P. Fougères, I. Gentil, F. Malrieu, C. Roberto, and G. Scheffer, Sur les inégalités de Sobolev logarithmiques, Panoramas et Synthèses, 10, Société Mathématique de France, 2000. 
[2] D. Bakry, L'hypercontractivité et son utilisation en théorie des semigroupes, in 'Lectures on probability theory' (Saint-Flour, 1992), Lecture Notes in Math., 1581, Springer, Berlin, 1994, 1-114.

[3] D. Bakry and M. Émery, Diffusions hypercontractives, in 'Séminaire de probabilités XIX', Lecture Notes in Math., 1123, Springer, Berlin, 1985, 177-206.

[4] E. Bell, Men of Mathematics, Simon and Schuster, 1937.

[5] Y. Brenier, Polar factorization and monotone rearrangement of vector-valued functions, Comm. Pure Appl. Math., 44 (1991), 375-417.

[6] J. Cheeger, Differentiability of Lipschitz functions on metric measure spaces, Geom. Funct. Anal., 9 (1999), 428-517.

[7] J. Cheeger and T. Colding, Lower bounds on Ricci curvature and the almost rigidity of warped products, Ann. of Math., 144 (1996), 189-237.

[8] J. Cheeger and T. Colding, On the structure of spaces with Ricci curvature bounded below I, J. Differential Geom., 46 (1997), 37-74.

[9] J. Cheeger and T. Colding, On the structure of spaces with Ricci curvature bounded below II, J. Differential Geom., 54 (2000), 13-35.

[10] J. Cheeger and T. Colding, On the structure of spaces with Ricci curvature bounded below III, J. Differential Geom., 54 (2000), 37-74.

[11] D. Cordero-Erausquin, R. McCann, and M. Schmuckenschläger, A Riemannian interpolation inequality à la Borell, Brascamp and Lieb, Inv. Math., 146 (2001), 219-257.

[12] L. Evans and W. Gangbo, Differential equations methods for the Monge-Kantorovich mass transfer problem, Mem. Amer. Math. Soc., 137(653) (1999).

[13] K. Fukaya, Collapsing of Riemannian manifolds and eigenvalues of Laplace operator, Invent. Math., 87 (1987), 517-547.

[14] M. Gromov, Metric structures for Riemannian and non-Riemannian spaces, Progress in Mathematics, 152, Birkhäuser, Boston, 1999.

[15] J. Heinonen, Lectures on analysis on metric spaces, Springer-Verlag, New York, 2001.

[16] R. Jordan, D. Kinderlehrer, and F. Otto, The variational formulation of the FokkerPlanck equation, SIAM J. Math. Anal., 29 (1998), 1-17.

[17] V. Kapovitch, Restrictions on collapsing with a lower sectional curvature bound, Math. Zeit., 249 (2005), 519-539.

[18] S. Keith, Modulus and the Poincaré inequality on metric measure spaces, Math. Z., 245 (2003), 255-292.

[19] P. Koskela, Upper gradients and Poincaré inequalities, in 'Lecture notes on analysis in metric spaces', Appunti Corsi Tenuti Docenti Sc., Scuola Norm. Sup., Pisa, 2000, $55-69$.

[20] A. Lichnerowicz, Géométrie des groupes de transformations, Travaux et Recherches Mathématiques III, Dunod, Paris, 1958.

[21] F. Liese and I. Vajda, Convex statistical distances, Teubner-Texte zur Mathematik, 95, BSB B. G. Teubner Verlagsgesellschaft, Leipzig, 1987.

[22] J. Lott, Some geometric properties of the Bakry-Émery-Ricci tensor, Comment. Math. Helv., 78 (2003), 865-883.

[23] J. Lott and C. Villani, Ricci curvature for metric-measure spaces via optimal transport, to appear, Annals of Math.

[24] J. Lott and C. Villani, Weak curvature conditions and functional inequalities, http://www.arxiv.org/abs/math.DG/0506481, 2005.

[25] R.J. McCann, A convexity principle for interacting gases, Adv. Math., 128 (1997), $153-179$.

[26] R.J. McCann, Polar factorization of maps on Riemannian manifolds, Geom. Funct. Anal., 11 (2001), 589-608.

[27] G. Monge, Mémoire sur la théorie des déblais et des remblais, Histoire de l'Académie Royale des Sciences de Paris, 1781, 666-704. 
[28] S.-I. Ohta, On measure contraction property of metric measure spaces, preprint, http://www.math.kyoto-u.ac.jp/ ${ }^{\sim}$ sohta/, 2005.

[29] F. Otto, The geometry of dissipative evolution equations: the porous medium equation, Comm. Partial Differential Equations, 26 (2001), 101-174.

[30] F. Otto and C. Villani, Generalization of an inequality by Talagrand, and links with the logarithmic Sobolev inequality, J. Funct. Anal., 173 (2000), 361-400.

[31] G. Perelman, The entropy formula for the Ricci flow and its geometric applications, http://www.arxiv.org/abs/math.DG/0211159, 2002.

[32] Z. Qian, Estimates for weighted volumes and applications, Quart. J. Math. Oxford, 48 (1997), 235-242.

[33] S. Rachev and L. Rüschendorf, A characterization of random variables with minimum $L^{2}$-distance, J. Multivariate Anal., 32 (1990), 48-54.

[34] M. von Renesse, On local Poincaré via transportation, preprint, http://www. arxiv.org/abs/math.MG/0505588, 2005.

[35] M.-K. von Renesse and K.-T. Sturm, Transport inequalities, gradient estimates and Ricci curvature, Comm. Pure Appl. Math., 68 (2005), 923-940.

[36] K.-T. Sturm, On the geometry of metric measure spaces, Acta Math., 196 (2006), 65-131.

[37] K.-T. Sturm, On the geometry of metric measure spaces II, Acta Math., 196 (2006), $133-177$.

[38] C. Villani, Topics in optimal transportation, Graduate Studies in Mathematics, 58, American Mathematical Society, Providence, 2003.

[39] C. Villani, Optimal transport, old and new, Springer-Verlag, to appear

Department of Mathematics, University of Michigan, Ann Arbor, MI 48109-1109, USA

E-mail address: lott@umich.edu 
